\title{
Ultrafast Electroabsorption at the Transition between Classical and Quantum Response
}

\author{
A. H. Chin, J. M. Bakker,* and J. Kono ${ }^{\dagger}$ \\ W. W. Hansen Experimental Physics Laboratory, Stanford University, Stanford, California 94305
}

(Received 10 March 2000)

\begin{abstract}
We report the first observation of unusually large induced absorption in semiconductors strongly driven by intense ultrashort midinfrared laser fields. This ultrafast electroabsorption has the largest extent below the band edge $(\sim 1 \mathrm{eV})$ ever observed, to our knowledge, which we interpret as being a manifestation of the dynamical Franz-Keldysh effect. The electroabsorption is observable when the ponderomotive potential is comparable to the photon energy of the applied field, i.e., when the applied field is at the transition between the classical and quantum regimes.
\end{abstract}

PACS numbers: 78.20.Jq, 42.50.Md, 78.30.Fs, 78.47.+p

Matter in the presence of an ac electric field of sufficient field strength exhibits phenomena that cannot be understood by treating the field as a small perturbation. For example, the ac (or optical) Stark effect in atoms [1] and semiconductors [2], which occurs in the presence of strong driving fields resonant (or nearly resonant) with electronic transitions, is better described by coupled light-matter states (or "dressed" states) than by perturbation theory. In the nonresonant (far from resonance) case, atoms under high-intensity laser excitation exhibit other nonperturbative phenomena, such as above-threshold ionization [3], high-order harmonic generation [4], and the laser-assisted photoelectric effect [5]. Here we report the first observation of ultrafast electroabsorption below the band edge in semiconductors strongly driven by intense midinfrared (MIR) pulses. This effect has the largest extent of electroabsorption ever observed, to our knowledge, and is another example of nonperturbative phenomena in the nonresonant case.

These nonresonant, nonperturbative phenomena are observed when the applied field is at the transition between the classical and quantum regimes, i.e., when interaction with the applied field cannot be treated by either neglecting photon effects or by considering only single photon effects. The measure of field strength defining these regimes is the time-averaged kinetic energy of an electron in a driving ac electric field, known as the ponderomotive potential. If the ponderomotive potential is comparable to or greater than the photon energy $(\hbar \omega)$ of the electric field and/or the relevant energy scale in the system (e.g., ionization energy), nonperturbative effects appear. For a driving ac electric field with vector potential $\vec{A}=\vec{A}_{0} e^{i \omega t}$, the ponderomotive potential (also known as the "wiggle" or "quiver" energy) is given by

$$
U_{p}=\frac{e^{2} A_{0}^{2}}{4 m c^{2}}=\left(\frac{2 \pi e^{2}}{m c}\right)\left(\frac{I}{\omega^{2}}\right),
$$

where $I$ is the intensity of the electric field at frequency $\omega$, and $m$ is the mass of the electron. When $U_{p} \ll \hbar \omega$, the applied field is approaching the extreme quantum limit and may be treated as a small perturbation. When $U_{p} \sim \hbar \omega$, the electric field of the driving light begins to significantly modify the electronic states in matter and cannot be treated perturbatively. This regime represents the transition between the classical and quantum regimes. Finally, when $U_{p} \gg \hbar \omega$ and $U_{p}$ is comparable to the relevant energy scale in matter $(\hbar \omega$ is less than the relevant energy scale of the system) the applied ac electric field behaves quasistatically (i.e., the system adiabatically follows the electric field) and photon effects are negligible. For example, ionization using laser fields behaves like multiphoton ionization when $U_{p} \ll \hbar \omega$ and like static electric field tunneling ionization when $U_{p}>E_{i p}>\hbar \omega$, where $E_{i p}$ is the ionization potential (i.e., when the Keldysh parameter [6] $\left.\gamma=\sqrt{E_{i p} / 2 U_{p}}<1\right)$. Thus, ponderomotive potential effects, i.e., effects at the transition between classical and quantum response, are most noticeable when $U_{p} \sim \hbar \omega$, but less than or comparable to the relevant energy scale in the system (e.g., the band gap in semiconductors).

Ponderomotive potential effects in semiconductors have been considered ever since the invention of the laser $[6,7]$. However, strong electronic absorption (the typical semiconductor band gap energy $E_{\text {gap }} \sim 1 \mathrm{eV}$ ) and competing high-intensity damage mechanisms [8] have made such observations impossible using traditional laser sources ( $E_{\text {photon }} \sim 1 \mathrm{eV} ; \lambda \sim 1 \mu \mathrm{m}$ ). Using longer wavelength light avoids the electronic absorption problem and avoids high-intensity damage mechanisms. In addition, the smaller effective masses $\left(\mathrm{m}^{*}\right)$ of carriers in semiconductors relative to the free electron mass $\left(m_{e}\right)$ makes the ponderomotive potential larger for a given laser intensity. Thus, the use of ultrashort MIR or far-infrared (FIR) driving fields in semiconductors permits the observation of the maximum possible ponderomotive potential effect, because $U_{p} \sim \hbar \omega<E_{\text {gap }}$ can be achieved while keeping multiphoton absorption of the intense driving field low.

Sources of intense MIR and FIR pulses that permit the study of ponderomotive potential effects in semiconductors have only recently become available. Using one such source, we observed ultrafast induced absorption below the band edge, which represents the first evidence of this aspect of the dynamical Franz-Keldysh effect (DFKE). Excitonic DFKE was recently observed in a quantum well semiconductor system using $1 \mathrm{THz}(4 \mathrm{meV})$ FIR 
radiation [9]. However, the virtual nature of the process was not demonstrated, and no induced absorption below the band edge was observed in the previous work. In order to observe ultrafast induced absorption below the band edge, we used an optical parametric amplifier (OPA) system with difference-frequency mixing as a source of tunable intense MIR pulses. The system produces MIR pulses $(\sim 3 \mu \mathrm{J} ; 1 \mathrm{kHz} ; \sim 1 \mathrm{ps})$ tunable from 3 to $10 \mu \mathrm{m}$ (see, e.g., [10]).

To study the DFKE, we measured the transmission near the band edge of bulk semiconductors using broadband light as a probe. The broadband light is produced by continuum generation in a sapphire plate (see, e.g., [10]), using the residual pump pulse $(800 \mathrm{~nm})$ after the OPA. The broadband probe is temporally overlapped with the MIR pump (or driving field) by changing the time delay (via the optical path length) of the MIR pump relative to the probe. The broadband probe and the MIR pump are focused onto the sample using an off-axis paraboloid, with the beams crossing at $\sim 10^{\circ}$. After passing through the sample, the spectrum of the broadband probe is dispersed using a grating monochromator and detected using a Si charge-coupled device camera (response between 1.1 and $\sim 3 \mathrm{eV}$ ). Spectra are obtained with the driving field at various time delays with respect to the probe and are subsequently normalized to the probe spectrum without the sample to obtain the absolute transmission (accurate to $\pm 5 \%$ ).

Shown in Fig. 1 is transmission data taken using a $3.5 \mu \mathrm{m}$ MIR driving field with $\sim 1$ ps pulse duration and $\sim 2 \times 10^{10} \mathrm{~W} / \mathrm{cm}^{2}$ peak incident intensity $\left(U_{p} \approx\right.$ $\hbar \omega=0.35 \mathrm{eV})$. Under these conditions, we observed a dramatic decrease in transmission that extends past $0.2 \mathrm{eV}$ below the band edge $\left(E_{\text {gap }}=1.4 \mathrm{eV}\right)$ of the GaAs sample [350 $\mu \mathrm{m}$ thick, semi-insulating, (100) surface]. The energy range over which the change occurs is larger than any observed electroabsorption using strong static fields [11] or energy shift due to intense laser pulses via

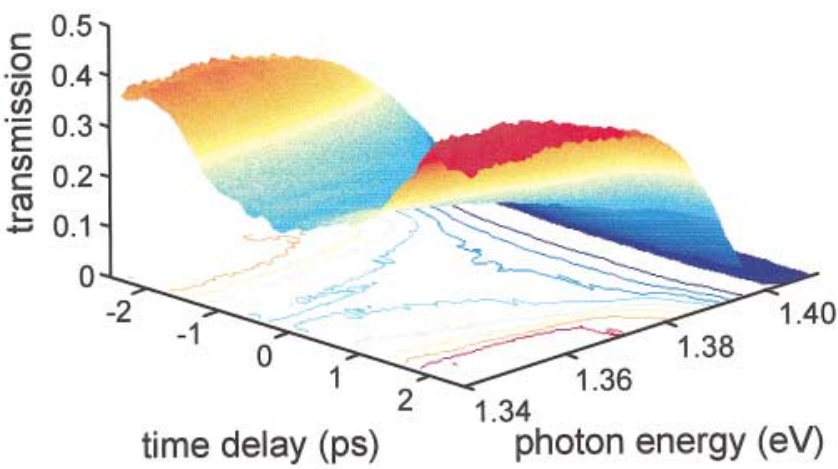

FIG. 1 (color). Transmission of a broadband probe pulse through GaAs (350 $\mu \mathrm{m}$ thick) as a function of time delay between the arrival of the intense $3.5 \mu \mathrm{m}$ MIR driving pulse and the broadband probe pulse (negative time delays correspond to MIR pulses arriving first). A contour plot is shown in the plane of the time delay and photon energy axes. the ac Stark effect [2]. This decrease in transmission, due to induced absorption, occurs only during the presence of the intense MIR pulse. This clearly demonstrates the virtual nature of the effect; i.e., no MIR excitation of carriers across the band gap and/or lattice-heating effects are involved. The effect is not found to depend on rotation of the sample in the surface plane, suggesting no dependence on crystal orientation. Shown in Fig. 2(a) is the wavelength dependence of the effect (pump and probe pulses overlapped) in GaAs, comparing the $3.5 \mu \mathrm{m}$ $\left(U_{p} \approx \hbar \omega=0.35 \mathrm{eV}\right)$ case and the $6.2 \mu \mathrm{m}$ case with $\sim 3 \times 10^{9} \mathrm{~W} / \mathrm{cm}^{2}$ peak incident intensity $\left(U_{p} \approx \hbar \omega=\right.$ $0.2 \mathrm{eV}$ ). Here we observe an effect in the $6.2 \mu \mathrm{m}$ case that is comparable to the $3.5 \mu \mathrm{m}$ case, with the extent below the band edge being comparable to the MIR photon energy. Shown in Fig. 2(b) is the intensity dependence of the effect in GaAs using $3.5 \mu \mathrm{m}$ MIR driving pulses, which is consistent with an absorption

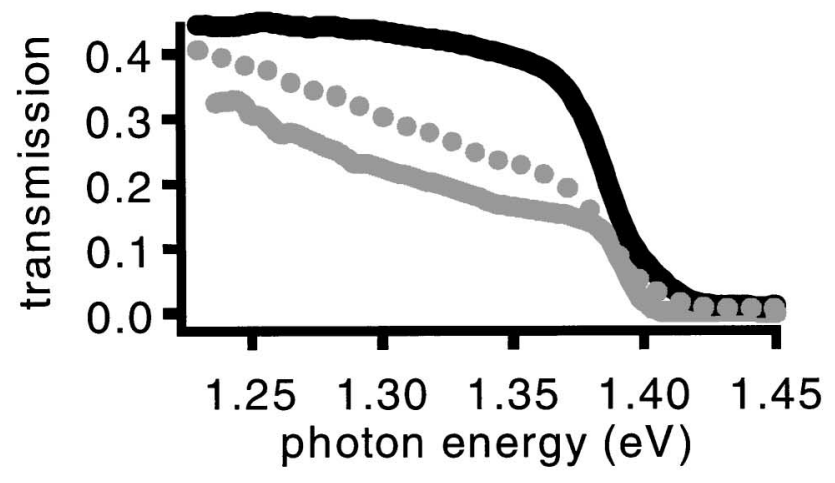

(a)

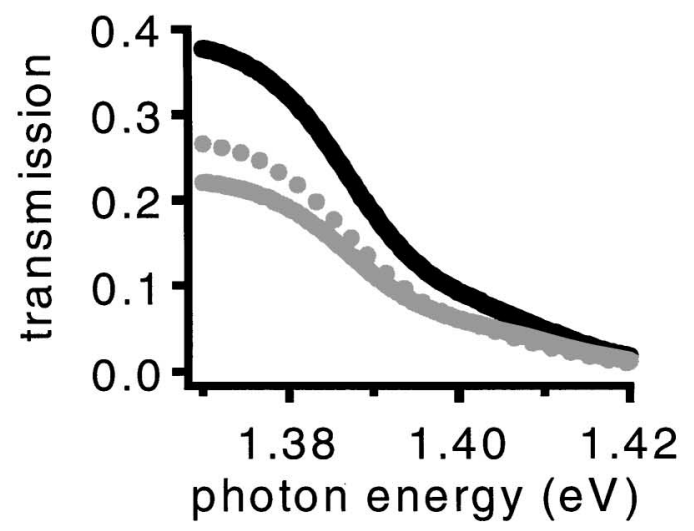

(b)

FIG. 2. (a) Wavelength dependence of electroabsorption in GaAs (350 $\mu \mathrm{m}$ thick): transmission below the band edge with $U_{p} \approx \hbar \omega$ using $3.5 \mu \mathrm{m}$ MIR driving pulse (gray line), with $U_{p} \approx \hbar \omega$ using $6.2 \mu \mathrm{m}$ MIR driving pulse (gray circles), and with no driving pulse (black line). (b) Intensity dependence of electroabsorption in GaAs (350 $\mu \mathrm{m}$ thick) using $3.5 \mu \mathrm{m}$ MIR driving pulses: transmission below the band edge at intensity $I_{0}$ (with $U_{p} \approx \hbar \omega$ ) (gray line), $I_{0} / 2$ (gray circles), and with no driving pulse (black line). 
coefficient that is linearly dependent on the driving field intensity. Finally, the effect is not specific to GaAs, as we also observed induced absorption in polycrystalline $\mathrm{ZnSe}$ $\left(E_{\text {gap }}=2.7 \mathrm{eV}\right)$ and crystalline ZnTe $\left(E_{\text {gap }}=2.3 \mathrm{eV}\right)$. The induced absorption in $\mathrm{ZnSe}$ ( $3 \mathrm{~mm}$ thick) with and without the presence of a $3.5 \mu \mathrm{m}$ MIR driving field is shown in Fig. 3. Here the large chirp present in the broadband probe over the frequency range considered $(\sim 2.5 \mathrm{ps})$ requires accounting for the chirp in the data processing but does not affect the interpretation of the effect. Our measurements clearly demonstrate the large extent $(\sim 1 \mathrm{eV}$ below the band edge) of the induced absorption, which far exceeds any previously observed extent of induced absorption in semiconductors.

We attribute the observed ultrafast electroabsorption to the dynamical Franz-Keldysh effect. Since the (staticfield) Franz-Keldysh effect $\left(U_{p} \gg \hbar \omega\right)$ can be thought of as a tunneling assisted transition [12,13], the DFKE $\left(U_{p} \sim \hbar \omega\right)$ may be intuitively thought of as the point where the tunneling time is comparable to the period of the applied electric field. Because the conduction band states are analogous to free electrons, an analogy can be made between the energy gap in a semiconductor and the ionization potential in a gas. Consequently, a quantitative description of the DFKE may be constructed in terms of effective mass Bloch-Volkov wave functions $[7,14]$ that are analogous to Volkov wave functions that represent free electrons in an electromagnetic field. Using this perspective, the conduction band wave function modified by a linearly polarized strong driving field $\vec{A}=\vec{A}_{0} e^{i \omega t}$ is given by the effective mass Bloch-Volkov wave function [14]

$$
\psi(\vec{r}, t)=\frac{1}{\sqrt{N_{0}}} u_{c, \vec{k}}(\vec{r}) \sum_{n=-\infty}^{\infty} e^{i n \omega t} e^{i \vec{k} \cdot \vec{r}} e^{-i E_{c} t / \hbar} J_{n}(u, \nu),
$$

where we utilize the generalized Bessel function notation [15] $J_{n}(u, \nu)$ and where $u_{c, \vec{k}}(\vec{r})$ is the periodic portion of the Bloch wave function, $E_{c}=\left(\hbar^{2} k^{2} / 2 m_{c}\right)+U_{p}$, $u=e \vec{A}_{0} \cdot \vec{k} / c m_{c} \omega, \nu=-U_{p} / 2 \hbar \omega, m_{c}$ is the conduction band effective mass, and $N_{0}$ is the number of unit cells in the crystal. As seen from Eq. (2), the modified conduction band can be considered to consist of replicas $(n \neq 0)$ of the original conduction band $(n=0)$, each separated by the driving field photon energy. This modification is the manifestation of the combined classical and quantum character of the response in this strongly driven regime, i.e., where $U_{p} \sim \hbar \omega$. These states in the modified conduction band are similar to dressed states in the resonant case, e.g., in the ac Stark effect. Transitions from the valence band to "dressed states" in this modified conduction band are weighted by a function of the driving field $[14,16]$. We assume the valence band is unaffected by the driving field because of the larger effective mass of holes, as well as the larger binding energy of the valence band relative to conduction band. The DFKE

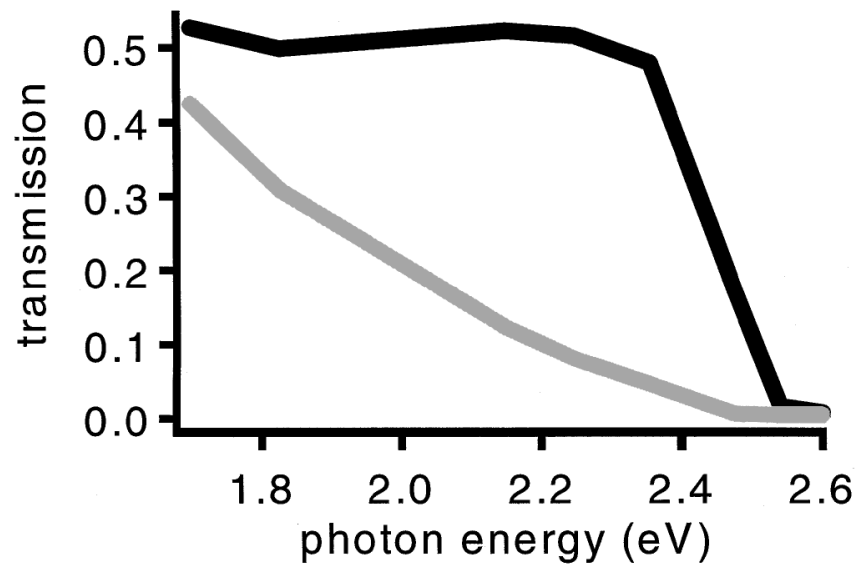

FIG. 3. Transmission below the band edge of $\mathrm{ZnSe}(3 \mathrm{~mm}$ thick) with (gray line) and without (black line) an intense $3.5 \mu \mathrm{m}$ MIR driving field with $U_{p} \approx \hbar \omega$.

cannot be understood by treating the applied strong laser light as a classical field (as in the static-field FranzKeldysh effect) or by single photon interactions (extreme quantum limit). Thus, the DFKE is an example of phenomena at the transition between classical and quantum response.

The existing models predict an induced absorption with an absorption coefficient that is linear with the ponderomotive potential (or the driving field intensity) and with an extent that is on the order of the ponderomotive potential (or photon energy, since $U_{p} \sim \hbar \omega$ ) below the band edge [7,17-19]. Both of these predictions are consistent with our data. Using a model based on the Bloch-Volkov wave functions and the theory of $x$-ray absorption in gases in the presence of a strong laser field [16], we simulated the data in GaAs driven by a $3.5 \mu \mathrm{m}$ MIR driving field (see Fig. 4). The theory assumes that only a $150 \mu \mathrm{m}$ thick part of the GaAs (the estimated overlap between the pump and probe beams) is affected by a field with an intensity (inside the sample) of $5 \times 10^{9} \mathrm{~W} / \mathrm{cm}^{2}$. In addition, the intrinsic absorption below the band edge, due to impurity absorption, phonon-assisted absorption (Urbach tail), etc., is accounted for by using the experimental data and assuming this remains unaffected by the MIR driving field. There is good agreement between our model describing the DFKE and our observations of ultrafast electroabsorption in semiconductors at the transition between classical and quantum response.

In addition to the DFKE, other phenomena at the transition between classical and quantum response in semiconductors may be studied. For example, phenomena analogous to those observed in gases driven by highintensity laser fields, such as above-threshold ionization [3], high-order harmonic generation [4], and the laser-assisted photoelectric effect [5], may be studied in semiconductors. Thus, semiconductors provide an alternative to gases excited by high-intensity laser pulses 


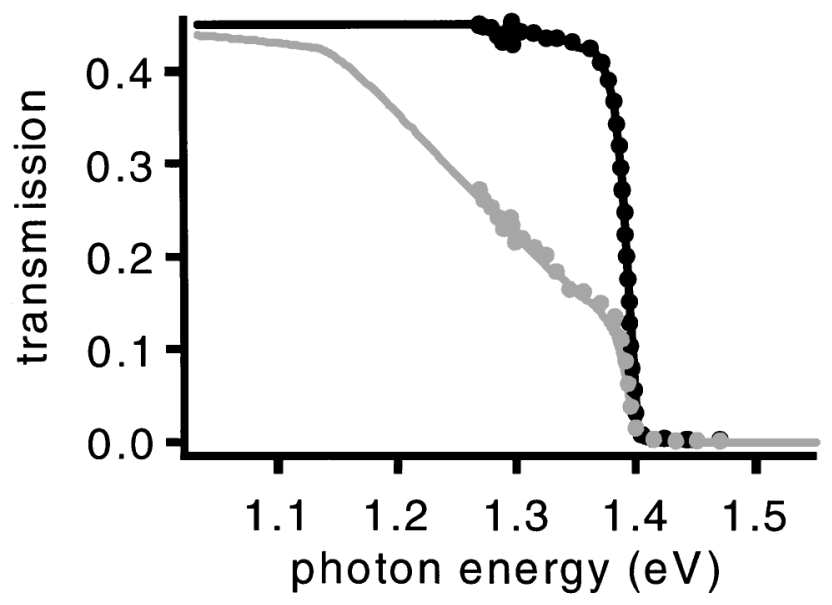

FIG. 4. Comparison of theory (solid lines) and experimental data (circles) in GaAs using $3.5 \mu \mathrm{m}$ MIR driving pulses for no field (black circles) and with $U_{p} \approx \hbar \omega$ (gray circles).

for studying ponderomotive potential phenomena. In addition, phenomena that cannot be observed in gases may be evident in semiconductors. For example, the effect of quantum confinement on ponderomotive potential effects [20] or the explicit coupling of the spatially periodic potential of the solid to the temporally periodic potential imposed by a strong electric field [17,21] may be observed.

Besides the scientific interest, our measurements may have an impact on future technological development. The desire for increased bandwidth in telecommunications will eventually lead to the development of ultrafast optical modulators operating at frequencies in the terahertz $(\mathrm{THz})$ range. Because of the large electro-optic modulation of near-infrared light using MIR pulses, one may use the DFKE using MIR lasers as a basis for an ultrafast optical modulator. Unfortunately, practical devices await the development of MIR laser technology for miniaturization. Alternatively, electronics may be developed that are able to apply strong fields to electro-optic devices at $\mathrm{THz}$ frequencies. However, as electronics and photonics approach $\mathrm{THz}$ frequencies, the transition between classical and quantum behavior will become important. Consider the case of GaAs (with effective mass of electrons, $m^{*}=0.07 m_{e}$ ) in the presence of a field with $\sim 1 \mathrm{THz}$ frequency and peak electric field $\sim 10^{4} \mathrm{~V} / \mathrm{cm}$, a typical field that can be applied in devices $(\sim 1 \mathrm{~V}$ over $\sim 1 \mu \mathrm{m}$ feature size). Under such a field in GaAs, $U_{p} \approx \hbar \omega=4 \mathrm{meV}$. Thus, the transition between classical and quantum response needs to be accounted for when considering the use of some of the current electrooptic technology at higher frequencies.

In conclusion, we have made the first observation of ultrafast electroabsorption below the band edge in semiconductors due to the dynamical Franz-Keldysh effect. The observed ultrafast absorption induced by intense MIR pulses has an extent below the band gap that is the largest ever observed, to our knowledge. The DFKE occurs when the strong applied field is at the transition between classical and quantum behavior, i.e., when the ponderomotive potential is comparable to the photon energy. The observation of other new phenomena like the DFKE involving the interplay between a spatially periodic potential and a temporally periodic potential may result from studies in the regime between classical and quantum response in semiconductors. In addition, as electronics continue to operate at higher frequencies and the technology gap between electronics and photonics closes, effects at the transition between classical and quantum response like the DFKE will have to be considered.

We thank T. Kimura for his help with the setup of the laboratory, H. A. Schwettman for his support, and T.E. Glover, K. Johnsen, D. S. Citrin, A. Liu, C.Z. Ning, Y. Kayanuma, A. Imamoglu, and S.E. Harris for enlightening discussions. We gratefully acknowledge support from NSF Grant No. DMR-9970962, No. ONR N00014-94-1-1024, the Japan Science and Technology Corporation PRESTO Program, and the NEDO International Joint Research Grant Program.

*Present address: FOM Institute for Plasma Physics Rijnhuizen, Edisonbaan 14, NL-3439 MN Nieuwegein, The Netherlands.

†Present address: Department of Electrical and Computer Engineering, Rice University, Houston, TX 77005.

[1] S. H. Autler and C. H. Townes, Phys. Rev. 100, 703 (1955).

[2] A. Mysyrowicz et al., Phys. Rev. Lett. 56, 2748 (1986).

[3] P. H. Bucksbaum, J. Opt. Soc. Am. B 4, 760 (1987).

[4] A. Rundquist et al., Science 280, 1412 (1998).

[5] T. E. Glover et al., Phys. Rev. Lett. 76, 2468 (1996).

[6] L. V. Keldysh, Sov. Phys. JETP 20, 1307 (1965).

[7] Y. Yacoby, Phys. Rev. 169, 610 (1968).

[8] B. C. Stuart et al., Phys. Rev. Lett. 74, 2248 (1995).

[9] K. B. Nordstrom et al., Phys. Rev. Lett. 81, 457 (1998).

[10] M. K. Reed and M. K. Steiner-Shepard, IEEE J. Quantum Electron. 32, 1273 (1996).

[11] D. A. B. Miller et al., Phys. Rev. B 32, 1043 (1985).

[12] D. A. B. Miller et al., Phys. Rev. B 33, 6976 (1986).

[13] W. Franz, Z. Naturforsch. 13A, 484 (1958); L. V. Keldysh, Sov. Phys. JETP 34, 788 (1958).

[14] H. D. Jones and H.R. Reiss, Phys. Rev. B 16, 2466 (1977).

[15] H. R. Reiss, Phys. Rev. A 22, 1786 (1980).

[16] M. Jain and N. Tzoar, Phys. Rev. A 15, 147 (1977).

[17] L. C. M. Miranda, Solid State Commun. 45, 783 (1983); Yu. I. Balkarei and E. M. Epshtein, Sov. Phys. Solid State 15, 641 (1973).

[18] O. A. C. Nunes, J. Appl. Phys. 58, 2102 (1985).

[19] K. Johnsen and A.-P. Jauho, Phys. Rev. B 57, 8860 (1998).

[20] D. S. Citrin and A. Maslov, Opt. Commun. 148, 187 (1998).

[21] F. H. M. Faisal and J.Z. Kaminski, Phys. Rev. A 56, 748 (1997). 